\title{
Cooperative Communications in Wireless Networks - A Requested Relaying Protocol
}

\author{
Tobias Renk, Clemens Kloeck, Dennis Burgkhardt, and Friedrich K. Jondral \\ Institut für Nachrichtentechnik \\ Universität Karlsruhe (TH), Germany \\ \{renk,kloeck,burgkhardt,fj\}@int.uni-karlsruhe.de
}

\begin{abstract}
In this paper we present a requested relaying protocol that helps mitigating multipath and shadowing effects in wireless networks. In our approach it is assumed that the destined mobile station has not been able to receive information from the base station and sends a request to surrounding terminals. Those terminals able to receive the request act as relays and help to transmit information from the base station to the destination. Performance analysis with respect to probability of decoding error and outage probability have been made with the results that both can be enormously improved by exploiting diversity. Additionally, order of diversity is defined and applied to our relaying approach as well.
\end{abstract}

\section{INTRODUCTION}

$\mathbf{M}$ ULTI-MEDIA communications demand for higher data rates and more reliable transmission schemes in future wireless networks. This demand cannot be accommodated by traditional techniques and hence new methodologies are necessary. In contrast to wired systems, wireless communication systems share an inherently finite resource, the radio frequency spectrum, where it is not possible to add new resources by simply appending new hardware equipment. One promising approach in that purpose are relay networks. Relay mechanisms use intermediate mobile stations for information transportation. The principle of relaying was introduced by van der Meulen [1] in 1968. Eleven years later, Cover and El Gamal [2] derived capacity theorems on the one relay case. Exploitation of cooperation among users is investigated in [3], [4], [5], and Laneman [6] gives a survey on conventional relay principles which we discuss in Section II.

Both, relaying and cooperation offer enormous performance enhancements in wireless communications. Various metrics for evaluation of performance are possible, for example capacity, reliability of communication, diversity gains, probability of decoding error, and outage probability [3], [4], [6], [7], [8].

In wireless communication systems, system performance is mainly limited by channel variations such as path loss, multipath fading, and shadowing. These issues motivate the design of relay networks. For example, due to the reduced distance between relay and destination (with respect to the distance source - destination), a relay can transmit at a lower power level which leads to energysavings. As a result of the fact that information is sent to the destination via several (independent) paths, effects of fading and shadowing can be mitigated to a certain extent. Nevertheless, if relaying is applied to nowadays mobile stations, relaying will surely produce a transmission delay since mobile stations are not yet able to receive and transmit simultaneously. For future systems and with respect to the keywords software-defined radio, cognitive radio, and multi-antenna (multi-receiver paths) this aspect produces no severe problems.

A major goal of relaying systems is the mitigation of outage events paired with energy-savings. This is the case for requested relaying systems that are in the scope of this paper. If the destination is not able to receive information from the source, i.e., base station, it will send a request for information to surrounding terminals. Since those terminals do only act as relays if they are requested for, this relaying principle achieves better energy-savings compared to other relaying schemes. Relaying does also lead to an increased coverage area. However, this is not the intention of this paper though geometry effects (positions of relays between source and destination) play an important role in relay networks.

The remainder of the paper is organized as follows. Section II gives a short overview of relaying principles which can be grouped into fixed protocols, adaptive protocols, and protocols with feedback (requested relaying). In Section III channel model and preliminaries are defined. Performance analysis with respect to probability of decoding error and outage probability are derived and discussed in Section IV. Finally, we give a short outlook on future research and conclude the paper with summarizing remarks.

\section{A SURVEY ON RELAY PRINCIPLES}

In this section we give a survey on conventional relaying principles [6], [8]. Relaying protocols can be grouped into three categories at first glance. These are fixed protocols, adaptive protocols, and protocols with feedback (requested relaying). Fixed protocols can further be divided into:

- amplify-and-forward: The relays receive information, amplify it, and transmit it again without prior decoding. In order to meet power constraints, relays must apply a specific amplifier gain that can depend on channel coefficients if the relays are able to estimate 


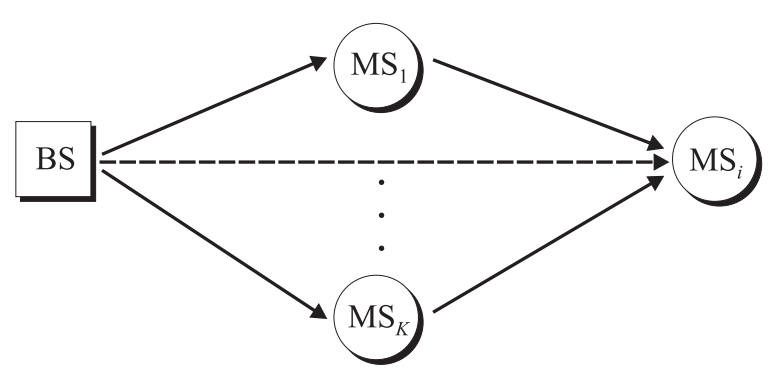

Fig. 1. Basic structure of relaying. A base station transmits information to $K$ mobile stations. The dashed line indicates that $\mathrm{MS}_{i}$ is not able to receive this information, e.g., due to shadowing. In this case an outage event at $\mathrm{MS}_{i}$ can be avoided thanks to relaying by the remaining mobile stations $\mathrm{MS}_{k}, k=1,2, \ldots, K, k \neq i$.

them. Drawback of amplify-and-forward transmission is noise enhancement.

- decode-and-forward: The relays receive information, decode it, and re-encode it before re-transmission. The degree of decoding at the relay may vary. For example, the relays could only perform modulation scheme dependent symbol-by-symbol decoding, whereas the destination performs full decoding. As the relays decode the information sequences, no noise enhancement is generated.

If channel state information (CSI) is available at the receivers (for example through pilot-assisted channel estimation), relays can adapt their transmissions to current channel conditions. This is the main attempt of adaptive protocols. A possible scheme could be, e.g., that a relay only transmits information to the destination if the channel gains between source and relay lie above a certain threshold. If not, the source re-transmits the information. The source gains knowledge about channel conditions through control information from the relays [9].

A major drawback of fixed and adaptive protocols is an inefficient use of degrees of freedom of the channel due to permanent relaying. This disadvantage can be overcome if the relays do not transmit relay information all the time, but only if they have received a request from the destination. This can either be a single bit that indicates successful reception of source information (in that case no relaying is necessary) or a longer bit-sequence that is only sent to the relays if relaying is wanted. In both cases reliable reception of the request information is expected. However, in the latter case, the bit-sequence may partly contain a known bitstructure which allows efficient channel estimation. In that case, a relay has the possibility to decide if relaying makes sense or if the channel conditions are too bad. This scheme improves spectral efficiency and saves energy at the relays.

\section{Channel Model and PReliminaries}

The basic structure of relaying systems can be seen in Fig. 1. A source (base station) transmits information to $K$ mobile stations. The dashed line indicates that the destination, $\mathrm{MS}_{i}$, is not able to receive the information from the base station. This has not to be the case in conventional relaying schemes, but is in the scope of this paper. The

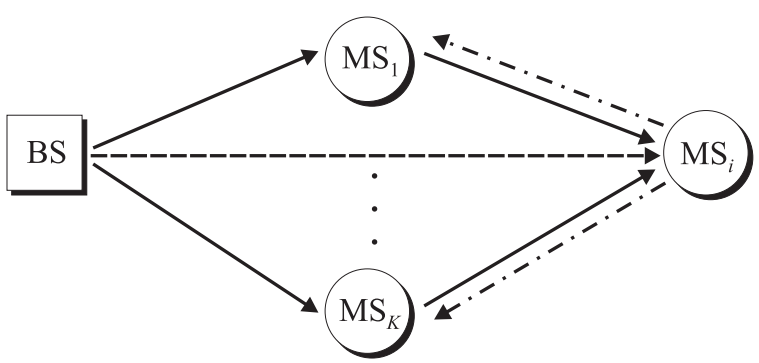

Fig. 2. Basic structure of requested relaying. A base station transmits information to $K$ mobile stations. The dashed line indicates that $\mathrm{MS}_{i}$ is not able to receive this information, e.g., due to shadowing. In this case $\mathrm{MS}_{i}$ sends out a request to surrounding mobile stations. If a remaining mobile station $\left(\mathrm{MS}_{k}, k=1,2, \ldots, K, k \neq i\right)$ receives this request correctly, it acts as relay.

remaining mobile stations, $\mathrm{MS}_{k}, k=1,2, \ldots, K, k \neq i$, of the network serve as relays. In order to use the degrees of freedom of the channel more efficiently and to improve spectral efficiency, we introduce requested relaying [10]. Fig. 2 illustrates the general structure where the dasheddotted line indicates the request. The relay is only sent if the mobile stations receive a request. If not, those mobile stations do not serve as relays which leads to energy savings.

For further investigations we define the following sets. Let $\mathcal{S}_{\mathrm{S}}$ denote the set of source terminals, $\mathcal{S}_{\mathrm{D}}$ denote the set of destination terminals, and $\mathcal{S}_{\mathrm{R}}$ denote the set of relay terminals. Hence, $\mathcal{S}_{\mathrm{S}}=\left\{\mathrm{BS}, \mathrm{MS}_{1}, \ldots, \mathrm{MS}_{K}\right\}, \mathcal{S}_{\mathrm{D}}=$ $\left\{\mathrm{MS}_{1}, \ldots, \mathrm{MS}_{K}\right\}$, and $\mathcal{S}_{\mathrm{R}}=\left\{\mathrm{MS}_{1}, \ldots, \mathrm{MS}_{K}\right\} \backslash\left\{\mathrm{MS}_{i}\right\}$, where $\mathrm{MS}_{i}$ is the requesting mobile station.

The overall transmission is divided into three transmission sequences. First, the transmission from the base station to the mobile stations, second, the request sent by $\mathrm{MS}_{i}$, and third, the relaying by the remaining mobile stations. The received signal vector at $\mathrm{MS}_{k}, k \in \mathcal{S}_{\mathrm{D}}$, after the first transmission sequence is given by

$$
\mathbf{y}_{k}(n)=\mathbf{H}_{k}(n) \mathbf{x}(n)+\mathbf{z}_{k}(n)
$$

where $\mathbf{y}_{k}=\left(y_{1}, \ldots, y_{N}\right)^{T}$ is the received signal, $\mathbf{x}_{k}=\left(x_{1}, \ldots, x_{N}\right)^{T}$ is the transmitted signal, and $\mathbf{z}_{k}=$ $\left(z_{1}, \ldots, z_{N}\right)^{T}$ is zero-mean independent identically distributed additive white Gaussian noise with one-sided power spectral density $\sigma_{z}^{2}=N_{0}$. The elements of the diagonal matrix $\mathbf{H}_{k}$ capture effects of path loss, shadowing, and flat fading, where $h_{0 k}$ is modeled as zero-mean, independent, circularly-symmetric complex Gaussian random variables ${ }^{1}$. Consequently, the magnitudes $\left|h_{0 k}\right|$ are Rayleigh distributed and $\left|h_{0 k}\right|^{2}$ is exponentially distributed with mean value $\sigma_{0 k}^{2}$ (generally, $\left|h_{0 k}\right|^{2}$ possesses a chisquare distribution). The phases $\arg \left(h_{0 k}\right)$ are uniformly distributed on $[0,2 \pi)$, however, we assume ideal phase recovery throughout the paper. Channel variations are considered to be constant over one transmission period (frame duration of length $N$ ). Therefore, the channel matrix can

\footnotetext{
${ }^{1}$ Throughout the paper the index $i k$ indicates transmission from $i$ to $k$, where 0 stands for the base station.
} 
be written as:

$$
\mathbf{H}_{k}=\left(\begin{array}{cccc}
\left|h_{0 k}\right| & 0 & \cdots & 0 \\
0 & \left|h_{0 k}\right| & \ddots & \vdots \\
\vdots & \ddots & \ddots & 0 \\
0 & \cdots & 0 & \left|h_{0 k}\right|
\end{array}\right) \in \mathbb{R}^{K \times N}
$$

Furthermore, it is assumed that $\left|h_{0 i}\right|=0$, which means that transmission from base station to $\mathrm{MS}_{i}$ fails. After one frame mobile station $\mathrm{MS}_{i}$ recognizes that it has no connection to the base station and sends a request. Those terminals that are able to receive the request then act as relays that send an estimation of the base station information $\mathbf{x}_{\text {relay }}=\hat{\mathbf{x}}(n)$ to the destination.

We expect some level of synchronization with respect to frame, carrier, and symbol synchronization. This must be ensured for the relay scheme to be effective. As can be seen in Fig. 2, we deploy a parallel relaying scheme with one intermediate hop. Note that this kind of parallel relaying scheme can be split up into a $1 \times K$ broadcast channel (transmission from the base station to the mobile stations) and a $(K-1) \times 1$ multiple access channel (from the relays to the destination) where it is assumed that all relays already have received the request correctly. For performance analysis we consider a symmetric network regime where all paths have identical properties. Moreover, we expect the destination to have $K-1$ antenna elements, each for a relay path, and the source to have $K-1$ antenna elements and full CSI. In [11] it is stated that for the case of full CSI at the transmitter waterfilling is the optimal power allocation. However, for our first analysis we assume that each antenna element transmits with equal power $P_{l}=P /(K-1)$ as every relay does. Thus, the total transmit power of the base station is $\sum_{l=1}^{K-1} P_{l}=P$.

\section{Performance Analysis}

\section{A. Error Performance}

The possibility of decoding error is introduced at each intermediate mobile station. Assuming that all transmission branches, and consequently the occurances of decoding errors on these branches, are independent, probability of decoding error for requested relaying with one requesting mobile station $\mathrm{MS}_{i}$ and $K-1$ relaying mobile stations can be expressed as

$$
p_{\mathrm{e}, 0 i}=\prod_{k \in \mathcal{S}_{\mathrm{R}}}\left[1-\left(1-p_{\mathrm{e}, 0 k}\right)\left(1-p_{\mathrm{e}, k i}\right)\right]
$$

where $p_{\mathrm{e}, 0 k}$ is the probability of decoding error from the base station to the relays and $p_{\mathrm{e}, k i}$ is the probability of decoding error from the relays to the destination, respectively (correct reception of the request is assumed).

For simulations we expect the average signal-to-noise ratio, $\overline{\gamma_{i k}}$, to be equal on every channel path. This yields

$$
\overline{\gamma_{i k}}=\mathcal{E}\left\{\alpha_{i k}^{2}\right\} \frac{P_{i}}{N_{0}}=\overline{\alpha^{2}} \frac{P}{(K-1) \cdot N_{0}}=\bar{\gamma},
$$

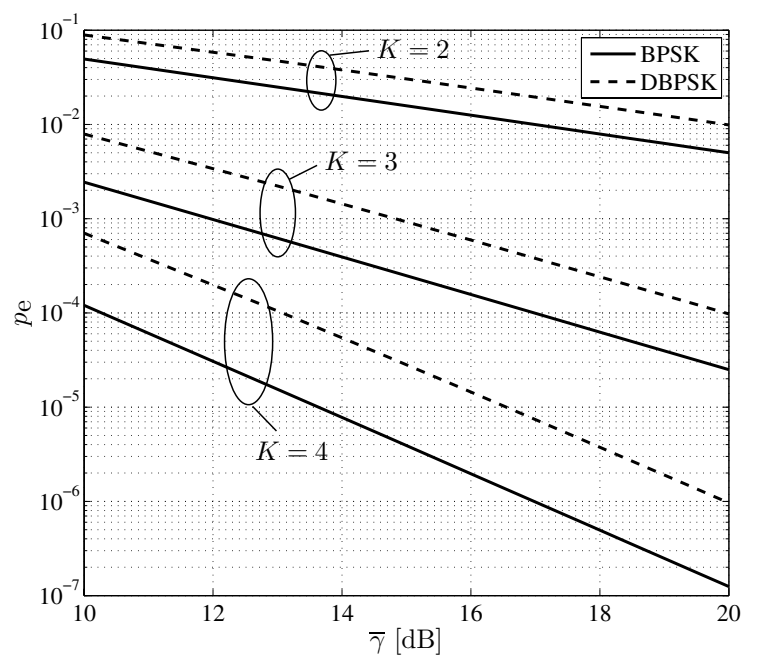

Fig. 3. Error probabilities for different numbers of relays $(K=2,3,4)$ in a Rayleigh fading environment when BPSK and DBPSK are used. For both modulation schemes error probability decreases exponentially with an increasing number of relays. The described scenario is a best case consideration for requested relaying as it is assumed that all relays have received the request correctly.

where $\mathcal{E}\{\cdot\}$ is the expectation operator. In Rayleigh fading environments and if binary phase shift keying (BPSK) modulation is applied, probability of decoding error can be approximated as [12]:

$$
p_{\mathrm{e}}=\left[1-\left(\frac{4 \bar{\gamma}-1}{4 \bar{\gamma}}\right)^{2}\right]^{(K-1)}
$$

For differential BPSK (DBPSK) error probability is given by [12]

$$
p_{\mathrm{e}}=\left[1-\left(\frac{1+2 \bar{\gamma}}{2(1+\bar{\gamma})}\right)^{2}\right]^{(K-1)} .
$$

Fig. 3 illustrates probability of decoding error for different numbers of relays ( $K=2,3,4)^{2}$. For both modulation schemes error probability decreases exponentially with the increasing number of relays. Due to this aspect, the gap between DBPSK and BPSK gets bigger with an increasing number of relays $\left(\propto 2^{(K-1)}\right)$. The described scenario is a best case consideration for requested relaying as it is assumed that all relays have received the request correctly. A typical characteristic of Rayleigh fading channels is the inverse algebraic relation between error probability and signal-to-noise ratio which expresses as a linear decrease of $p_{\mathrm{e}}$ as $\bar{\gamma}$ [dB] increases [12], [13].

\section{B. Outage Probability}

Outage probability describes the probability that the signal-to-noise ratio $\gamma$ at a mobile station falls below a required signal-to-noise ratio $\gamma_{\text {th }}$ that ensures reliable communication. For the following paragraph we assume that all relays have already received the information they are to

\footnotetext{
${ }^{2}$ Note that the number of relays is $K-1=\left|\mathcal{S}_{\mathrm{R}}\right|$, where $|\cdot|$ denotes the cardinal number of a set.
} 
send. This assumption makes sense insofar as a mobile station that has no knowledge about the information to send cannot serve as relay. To sum up, an outage event occurs if the request fails or the relay after having received a request correctly fails.

If we assume that the probability of an outage event is the same on every path (request as well as relay), $p_{\mathrm{o}}$, outage probability in the case of requested relaying where $K-1$ mobile stations serve as relays is given by:

$$
p_{\text {out }}=p_{\mathrm{o}}^{K-1}\left(2-p_{\mathrm{o}}\right)^{K-1}
$$

The proof of (7) can be found in the Appendix.

The following derivations expect a Rayleigh fading environment. If each path has an instantaneous signal-tonoise ratio $\gamma_{i k}$, then the probability density function of $\gamma_{i k}$ is [12]

$$
\operatorname{pdf}\left(\gamma_{i k}\right)=\frac{1}{\bar{\gamma}} \cdot \exp \left(-\frac{\gamma_{i k}}{\bar{\gamma}}\right)
$$

where it is assumed that each path has the same mean signal-to-noise ratio $\bar{\gamma}$ (see (4)). The probability that a single path has a signal-to-noise ratio below a given threshold value can then be expressed as:

$$
\begin{aligned}
p_{\mathrm{o}} & =\operatorname{Pr}\left[\gamma_{i k} \leq \gamma_{\mathrm{th}}\right] \\
& =\int_{-\infty}^{\gamma_{\mathrm{th}}} \operatorname{pdf}\left(\gamma_{i k}\right) d \gamma_{i k} \\
& =1-\exp \left(-\frac{\gamma_{\mathrm{th}}}{\bar{\gamma}}\right)
\end{aligned}
$$

Fig. 4 illustrates outage probability $p_{\text {out }}$ as a function of $\gamma_{\mathrm{th}} / \bar{\gamma}[\mathrm{dB}]$. As parameter the number of mobile stations in the network has been employed $(K=2,3,4)$. As expected, outage probability decreases as the number of relays increases. This fact is due to diversity gains (see Subsection IV-C).

In [14] the minimum required signal-to-noise ratio is denoted as

$$
\gamma_{\mathrm{th}}=b \cdot\left(2^{\mathcal{C}}-1\right)
$$

with $b>1$ and probably 3 for practical implementations. In (10), $\mathcal{C}=R / W[\mathrm{bit} / \mathrm{s} / \mathrm{Hz}]$ is the spectral efficiency, $R[\mathrm{bit} / \mathrm{s}]$ is the data rate, and $W[\mathrm{~Hz}]$ is the allocated bandwidth, respectively. The curve of $\gamma_{\mathrm{th}}[\mathrm{dB}]$ versus $\mathcal{C}$ is illustrated in Fig. 5. For ideal bandlimited additive white Gaussian noise channels, $b=1$, and a bandwidth that tends to infinity, we obtain the well-known maximum data rate that was first calculated by Claude E. Shannon [15]:

$$
R_{\max }=\lim _{W \rightarrow \infty} W \log \left(1+\gamma_{\mathrm{th}}(\mathcal{C})\right)
$$

Eventually, this leads to a required minimum signal-tonoise ratio per information bit for reliable communications of $\left(E_{b} / N_{0}\right)_{\min }=\ln 2=-1.59 \mathrm{~dB}$.

\section{Diversity Order}

The definition of diversity order is given in [6] as:

$$
d(\mathcal{C}) \triangleq-\lim _{\gamma \rightarrow \infty} \frac{\log p_{\text {out }}(\gamma, \mathcal{C})}{\log (\gamma)}
$$

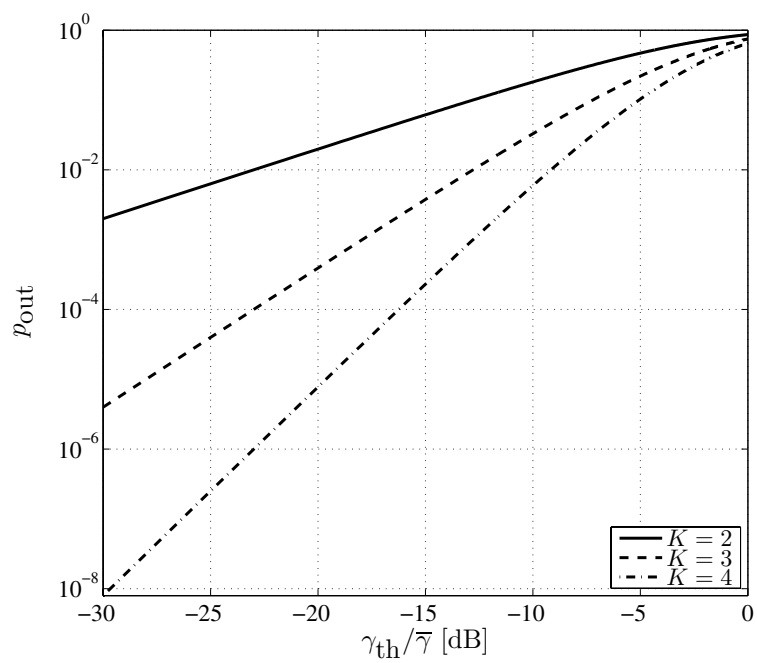

Fig. 4. Outage probability $p_{\text {out }}$ as a function of $\gamma_{\mathrm{th}} / \bar{\gamma}[\mathrm{dB}]$ for parameter $K=2,3,4$. Outage probability decreases as the number of relays $(K-1)$ increases due to diversity gains.

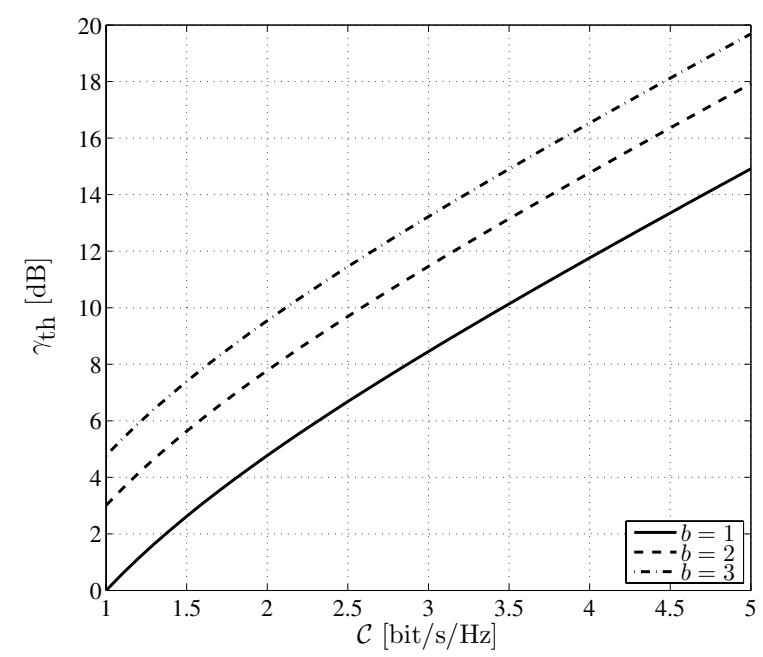

Fig. 5. Minimum required signal-to-noise ratio for reliable communications, $\gamma_{\text {th }}[\mathrm{dB}]$, versus spectral efficiency $\mathcal{C}[\mathrm{bit} / \mathrm{s} / \mathrm{Hz}]$. The parameter $b$ has been set to $b=1,2,3$, where $b=1$ represents the theoretical limit of Shannon's channel capacity and $b=3$ is a value for practical implementations.

We can see that diversity in general depends on spectral efficiency. However, this dependency vanishes due to limit calculations in our case. Applying (7) and (9) yields:

$$
\begin{aligned}
d & =-\lim _{\bar{\gamma} \rightarrow \infty} \frac{(K-1) \cdot\left[\log \left(p_{\mathrm{o}}\right)+\log \left(2-p_{\mathrm{o}}\right)\right]}{\log \bar{\gamma}} \\
& =K-1
\end{aligned}
$$

It can be seen that diversity order directly depends on the number of relays in the network.

\section{OutLook}

In this paper we expected synchronization between the base station and the mobile stations. Indeed, synchronization plays an important role in relay networks and must 
be considered thoroughly in future research. Today, mobile stations consist of one receiving path. However, if we allow a mobile station to send a request to surrounding stations while they are receiving information from the base station, then at least two communication paths are necessary. In addition, relays will not be transmitted simultaneously as this depends on the position of the surrounding relays relative to the requesting mobile station. Consequently, the mobile station that receives the relays must have the ability to appropriately combine the relay paths.

One major point is the structure of information of the request and the relays. For example, the request may only contain information which asks for relaying. Then the relays transmit as soon as they receive the request without any further processing. Drawback of this approach is that relay information is sent even if the channel is weak and no cooperation is exploited. Another possibility is that the request contains a known pilot sequence that is used in the relays for channel estimation. Then, a relay is only sent if the channel is good enough (e.g., $p_{\mathrm{e}}<p_{\mathrm{e}, \max }$ or $\gamma>\gamma_{\min }$ ) or a relay is always sent but the transmission parameters (data rate, transmit power, coding, etc.) are adapted accordingly. Moreover, adjacently located relays could cluster in order to send relay information in a virtual multiple-input multiple-output manner thus being able to deploy efficient space-time coding techniques.

Effects of geometry that manifest as utilization regions must also be included in further studies. Note that geometry constellations certainly come along with corresponding path loss considerations.

\section{CONCLUDING REMARKS}

In the near future it will become mandatory to create new techniques that are able to satisfy the demand for higher data rates and more reliable transmission schemes for multi-media communications. In this paper we presented a requested relaying protocol that helps mitigating multipath and shadowing effects in wireless networks. We assumed that the destination has not been able to receive information from the base station. Therefore, it sends out a request to surrounding mobile stations. Those mobile stations able to receive the request serve as relays. As we only have one intermediate station, the parallel transmission scheme can simply be split up into a broadcast and a multiple access channel.

We showed that both probability of decoding error as well as outage probability can be improved by exploiting diversity. Investigations on the minimum required signalto-noise ratio and order of diversity have also been made with the result that order of diversity increases linearly with the number of applied relays.

\section{APPENDIX}

Equation (7) can be proved by using a combinatorial ansatz.

Proof: Outage probability can be expressed as

$$
p_{\text {out }}=\sum_{k=0}^{K-1}\left(\begin{array}{c}
K-1 \\
k
\end{array}\right) p_{\mathrm{o}}^{k}\left(1-p_{\mathrm{o}}\right)^{K-1-k} p_{\mathrm{o}}^{K-1-k} \text {. }
$$

Since $p_{\mathrm{O}}$ is the same on every path we do not need to distinguish which relay is actually requesting as long as it is only one. Equation (14) can be written as:

$$
p_{\text {out }}=p_{\mathrm{o}}^{K-1} \sum_{k=0}^{K-1}\left(\begin{array}{c}
K-1 \\
k
\end{array}\right)\left(1-p_{\mathrm{o}}\right)^{K-1-k}
$$

If we compare (14) to the binomial theorem

$$
\sum_{k=0}^{n}\left(\begin{array}{l}
n \\
k
\end{array}\right) a^{n-k} b^{k}=(a+b)^{n}
$$

and substitute $n \triangleq K-1, a \triangleq 1-p_{\mathrm{o}}$, and $b \triangleq 1$ this yields:

$$
p_{\text {out }}=p_{\mathrm{o}}^{K-1}\left(2-p_{\mathrm{o}}\right)^{K-1}
$$

\section{ACKNOWLEDGMENT}

This work was performed in project $E^{2} \mathrm{R}$ II which has received research funding from the Community's Sixth Framework program. This paper reflects only the authors' views and the Community is not liable for any use that may be made of the information contained therein. The contributions of colleagues from $\mathrm{E}^{2} \mathrm{R}$ II consortium are hereby acknowledged.

\section{REFERENCES}

[1] E. van der Meulen, "Transmission of information in a t-terminal discrete memoryless channel," Department of Statistics, University of California, Berkeley, CA, Technical Report, 1968.

[2] T. Cover and A. El Gamal, "Capacity theorems for the relay channel," IEEE Transactions on Information Theory, vol. 25, no. 5, pp. 572-584, September 1979.

[3] A. Sendonaris, E. Erkip, and B. Aazhang, "User cooperation diversity - part I: System description," IEEE Communications Magazine, vol. 51, pp. 1927-1938, 2003.

[4] — , "User cooperation diversity - part II: Implementation aspects and performance analysis," IEEE Communications Magazine, vol. 51, pp. 1939-1948, 2003.

[5] G. Kramer, M. Gastpar, and P. Gupta, "Cooperative strategies and capacity theorems for relay networks," IEEE Transactions on Information Theory, vol. 51, no. 9, pp. 3037-3063, September 2005.

[6] J. Laneman, D. Tse, and G. Wornell, "Cooperative diversity in wireless networks: Efficient protocols and outage behavior," IEEE Transactions on Information Theory, vol. 50, no. 12, pp. 30623080, December 2004.

[7] A. Høst-Madsen and J. Zhang, "Capacity bounds and power allocation for wireless relay channels," IEEE Transactions on Information Theory, vol. 51, no. 6, pp. 2020-2040, June 2005.

[8] P. Herhold, E. Zimmermann, and G. Fettweis, "Cooperative multihop transmission in wireless networks," Journal on Computer Networks, vol. 49, no. 3, pp. 299-324, October 2005.

[9] E. Zimmermann, P. Herhold, and G. Fettweis, "On the performance of cooperative diversity protocols in practical wireless systems," 58th IEEE Vehicular Technology Conference, Orlando, Florida, USA, October 2003.

[10] T. Renk, C. Kloeck, H. Jaekel, and F. Jondral, "A new cognitive pilot channel concept based on multi-hop networks," Wireless World Research Forum 17, Heidelberg, Germany, November 2006.

[11] E. Telatar, "Capacity of multi-antenna gaussian channels," Bell Labs Journal, vol. 10, no. 6, November 1999.

[12] T. Rappaport, Wireless Communications: Principles \& Practice. New Jersey: Prentice Hall, 1996.

[13] M. Simon and M. Alouini, Digital Communication Over Fading Channels. New York: Wiley, 2000.

[14] T. Shepard, "Decentralized channel management in scalable multihop spread-spectrum packet radio networks," Ph.D. dissertation, Massachusetts Institute of Technology, July 1995.

[15] C. E. Shannon, "Communication in the presence of noise," Proc. Institute of Radio Engineers, vol. 37, no. 1, pp. 10-21, 1949. 